\title{
3. Die Hilfe zum Lebensunterhalt
}

\section{Laufende Unterstützungen}

Zwei Monate nach Muthesius' aufsehenerregendem Referat über eine staatliche Einkommenshilfe auf dem Fürsorgetag 1955 legte Gottschick seinem neuen Abteilungsleiter ein Konzept über die laufende Unterhaltsleistung im künftigen Fürsorgerecht vor. Zwar unter neuem Namen enthielt diese "Sozialunterstützung“ doch alle wesentlichen Elemente der traditionellen laufenden Fürsorgehilfen und sollte im Rahmen des neuen Bundesfürsorgegesetzes geregelt werden. ${ }^{212}$ Die Chancen für eine Umsetzung der Vorschläge von Muthesius standen also in der Sozialabteilung Anfang 1956 nach wie vor nicht günstig. Scheffler machte allerdings deutlich, daß der Hilfe zum Lebensunterhalt im neuen Fürsorgerecht ein Sonderstatus zugedacht war: Anders als die Individualhilfen werde sie „weitgehend schematisiert"; für die Individualhilfen kämen andere Grenzen der Hilfsbedürftigkeit in Betracht, und das „der Fürsorge noch anhaftende Odium“ sei „vor allem für die sonstigen Arten der Hilfe zu beseitigen“. ${ }^{213}$

Anfang September 1956 hielt Muthesius vor dem konservativen Gremium des DLT-Sozialausschusses einen Vortrag über „Grundsätzliche Fragen zur Neuordnung des Fürsorgerechts“, der in der Fachwelt beträchtliches Aufsehen erregte, da sich der DV-Vorsitzende noch deutlicher als bisher von den Grundlagen der traditionellen Fürsorge entfernte. Unter den Empfängern laufender Fürsorgeleistungen, so Muthesius, bildeten Sozialrentner und Rentenanwärter zwei Sondergruppen, die man nicht nach den Grundsätzen der Individualität und Subsidiarität betreuen könne, zumal das aus Sicht der Verwaltung oft vorteilhafte Individualprinzip für den Empfänger „auch eine Belastung darstellen“ könne. ${ }^{214}$ Die Ausgangsüberlegung, daß die Ergänzung anderweitiger Sozialleistungen nicht genuine Aufgabe der Fürsorge sei, war dabei kaum umstritten und wurde auch vom Innenminister im Bundeskabinett vertreten. ${ }^{215}$ Allerdings zeichnete sich im Herbst 1956 bereits ab, daß sich die mit der Rentenreform verknüpften diesbezüglichen Hoffnungen nicht so schnell erfüllen würden: Zwar würden neue Bemessungsgrundlagen und die geplante Dynamisierung viele Sozialrenten deutlich erhöhen; doch die Verschärfung des Äquivalenzprinzips begründete künftig den Verzicht auf einen festen „Grundbetrag“, so daß vor allem durch geringes Arbeitseinkommen verursachte Niedrigrenten trotz gewisser

212 Vgl. Anlage „Sozialunterstützung“ vom 10.1.1956 zu Abteilungsleitervorlage vom 4.2. 1956, sowie zwei Vermerke über die Besprechung mit dem Abteilungsleiter am 7.2.1956 nebst Anlage, BAK, B 106/9688. Das entsprach auch den Plänen des gerade aus dem Amt geschiedenen Wilhelm Kitz; vgl. Vermerk Referat V A 1 vom 27.7.1955, BAK, B $106 / 20652$.

213 Scheffler an Heusler etc. am 9.2.1956, Anlage; ähnlich u.a. vor den Länderwohlfahrtsministern am 29.5.1956, Kurzprotokoll, BAK, B 106/9789/2.

214 Manuskript des Vortrags vom 5. 9. 1956, BAK, B 106/9697 (Hervorhebung im Original).

215 Vgl. Vermerk Bangert über ein Gespräch mit Gottschick vom 25.5.1956, BAK, B $172 / 444-01 / 4$. 
Abfederungsmaßnahmen auch weiterhin von der Fürsorge zu ergänzen sein würden. ${ }^{216}$ Tatsächlich sank der Anteil der von der Fürsorge zusätzlich zu unterstützenden Sozialrentner nach der Rentenreform 1957 vorerst nur um ein Viertel. ${ }^{217}$

Isoliert blieb Muthesius allerdings mit seinem Lösungsvorschlag: Zusammengefaßt „in einem eigenen Rechtsinstitut“, dem „sozialen Ausgleich“, sollten unzureichende Renten durch örtliche Leistungen ergänzt und dabei ähnlich wie bei der - bedarfsabhängigen, aber mit gesetzlich definierten Beträgen arbeitenden Ausgleichsrente für Kriegsopfer verfahren werden. ${ }^{218}$ Finanziert werden sollte dies durch den Bund, um die Ergänzungsleistungen nicht „von der örtlichen Leistungsfähigkeit des Trägers abhängig zu machen“. Eine solche staatliche Sozialrentnerhilfe gab es seit 1952 im Saarland ${ }^{219}$.

Zwar stießen Muthesius' Beweggründe, hilfsbedürftigen Sozialrentnern individuelle Bedürftigkeitsprüfungen zu ersparen, auch im Beirats-Ausschuß auf Sympathie. ${ }^{220}$ Doch mit seinem Vorschlag setzte er sich zwischen alle Stühle: Kurt Jantz vom Arbeitsministerium erklärte, eine „Aufstockung von Rentenleistungen nach anderen als den der Rentenversicherung wesensgemäßen Prinzipien sei nicht vertretbar“221; tatsächlich hätte eine solche Übernahme beitragsunabhängiger Ergänzungsleistungen in die Sozialversicherung die gerade von ihm und seinem Minister forcierte Verstärkung des Äquivalenzprinzips konterkariert. Auch Walter Auerbach zitierte abwehrend den auf Walter Bogs zurückgehenden Grundsatz, wonach eine „weiche“, d.h. nicht auf einem Rechtsanspruch beruhende, $\mathrm{Zu}$ satzleistung eine eigentlich „harte“, d.h. aus einem eindeutigen Rechtsanspruch abgeleitete, Rentenleistung aus Sicht des Empfängers entwerte. ${ }^{222}$ Von seiten der Fürsorgeträger wurde u.a. eingewendet, daß dann „gerade diejenigen Personen aus der Betreuung der Fürsorge herausgenommen würden, die am ehesten geeignet wären, der Fürsorge das Odium des Lästigen zu nehmen“. ${ }^{223}$ Elsholz hatte auch prinzipielle Vorbehalte: Abgesehen davon, daß der Bund schon jetzt mit der Kriegsfolgenhilfe einen Teil der Aufstockungsleistungen trug, störte ihn der „sozialreformerische(n) Antrieb" solcher Maßnahmen, also die weitere Lockerung der Bindung von steuerfinanzierten Sozialleistungen an eine eng definierte Hilfs-

216 Vgl. ausführlich Hockerts, Entscheidungen, S.358ff., 400ff., 424. Hinzu kam, daß wieder eine Freilassung von Rententeilen eingeführt worden war und so die Fürsorgeträger nicht voll von den Rentenerhöhungen profitieren konnten.

217 Bei einem weiteren Viertel wurde die Ergänzung infolge der Rentenerhöhungen gekürzt; vgl. Wirtschaft und Statistik N.F. 10 (1958), S. 409; Oel, Fürsorge, S. 146.

218 Anders als bei den Vorarbeiten zum FÄG und zweifellos zum Unmut der meisten Fürsorgeexperten hielt Muthesius es jetzt auch für vertretbar, dabei teilweise den Weg der Nichtanrechnung bestimmter Sozialleistungen zu gehen, denn man habe ja inzwischen eingesehen, daß man mit Hilfe von Freilassungsvorschriften „ganz grossartig individualisieren kann“, Muthesius am 5.9.1956, BAK, B 106/9697.

219 Vgl. NDV 37 (1957), S. 33.

220 Vgl. Niederschrift über die Sitzung des Arbeitsausschusses für Fragen der Fürsorge am 6./7.4.1956, ADW, HGSt 6769.

221 Niederschrift über die Sitzung des Arbeitsausschusses am 21.2.1956, ebenda.

$222 \mathrm{Vgl}$. ebenda.

223 Petersen auf der Sitzung des Arbeitsausschusses am 6./7.4.1956, Niederschrift, ebenda. 
bedürftigkeit.224 Scheffler hingegen zeigte auf dem Fürsorgetag 1957 durchaus Sympathien für Muthesius' Überlegungen, resignierte aber vor dem Widerstand aus den Reihen der Rententräger. ${ }^{225}$

Muthesius' Bemühungen enthielten aber noch eine weitere, aus seinem Munde überraschende Komponente: Die laufenden Fürsorgeleistungen, die nicht andere Sozialleistungen ergänzten (also vor allem arbeits- bzw. rehabilitationsfähige Empfänger betrafen) solle man nicht mehr nach Richtsätzen bemessen, sondern zu bestimmten Arbeitseinkommen ins Verhältnis setzen, möglicherweise sogar für verschiedene Empfängergruppen zu verschiedenen Bezugseinkommen. ${ }^{226}$ Er begründete dies mit den Schwierigkeiten der Berechnung eines künstlichen Einkommens mittels Richtsatz/Warenkorb und stellte damit die von ihm selbst geleiteten, wegweisenden DV-Arbeiten der vergangenen beiden Jahre nachträglich in Frage. 227 Darüber hinaus könne durch diese Lohnrelation das vielbemühte Argument der Lähmung des Arbeitswillens ein für allemal entkräftet werden; hier warf Muthesius einen „Köder“ für die Landkreisvertreter aus, die ja vornehmlich mit den geringen Einkommen in strukturschwachen Landkreisen gegen eine stärkere Vereinheitlichung der laufenden Unterstützungsleistungen argumentierten. ${ }^{228}$ Muthesius erklärte es für durchaus denkbar, anstelle der „überholt[en]“ Einheitsfürsorge (!) „den Lebensstandard der uns Anvertrauten verschieden hoch anzusetzen“ (Hervorhebung im Original). Eine derart „differenzierte Fürsorge“ löse nicht nur „die Frage des unglücklichen Mehrbedarfs“, sondern sei auch „sozial gerecht".

Dieser Wechsel des Unterstützungsparameters kam in letzter Konsequenz einer Verabschiedung des gerade erst stärker qualifizierten fürsorgerischen Bedarfsprinzips gleich: Die Bemessung des Unterhalts für die laufenden Lebensbedürfnisse wäre dem kommunalen Ermessen endgültig genommen und weiter standardisiert worden. Darüber hinaus zog Muthesius Parallelen zur Rentenreform und kalkulierte damit zumindest implizit eine Steigerung auch der laufenden Leistungen zum Lebensunterhalt ein. ${ }^{229}$ Dieser offensichtlich beabsichtigte positive Effekt hing freilich entscheidend von der Auswahl der zugrundegelegten Arbeitslöhne ab: Die Koppelung an die aktuellen Durchschnittslöhne hätte bei weiterem Wirtschaftswachstum für die laufenden Fürsorgeleistungen eine automatische Dynamisierung bedeutet und damit auch dem Fürsorgeempfänger größere Teilhabemöglichkeiten eröffnet. Die Bindung an Niedriglöhne hingegen könnte noch gegenwärtige Richtsätze unterschreiten.

\footnotetext{
224 Elsholz, ebenda.

225 Vgl. Gerhard Scheffler, Neuordnung, S.22f.; ähnlich bereits auf einer internen Besprechung am 24.4.1957, Vermerk Referat V A 4, BAK, B 106/9789/2.

226 Muthesius vor dem DLT-Sozialausschuß am 5. 9.1956, Ms., BAK, B 106/9697.

227 Manchmal, so Muthesius, habe er „das Gefühl, unser kleines Buch ,Öffentliche Einkommenshilfe und Richtsatzpolitik' ist überhaupt vielleicht der Schwanengesang des Richtsatzsystems gewesen“, ebenda.

228 Vgl. Heisig, Armenpolitik, 1995, S. 141f.

229 Muthesius rekurrierte ausdrücklich auf die neue „Beweglichkeit“ der Renten, Muthesius am 5. 9.1956, Ms., BAK, B 106/9697.
} 
Nach anfangs durchaus positiver Reaktion des DLT-Sozialausschusses auf eine (niedrig-)lohnbezogene Fürsorgeleistung überwog dort schließlich doch die Furcht vor nach oben nivellierenden Auswirkungen einer automatischen Lohnkoppelung, war eine örtliche Besonderheiten berücksichtigende Differenzierung doch eher unwahrscheinlich. ${ }^{230}$ Hinzu kam die auch bei den Länderfürsorgereferenten vorhandene Sorge vor möglichen Rückkoppelungseffekten, die eine lohnbezogene, aber durch Rechtsanspruch gleichzeitig zur Bedarfsdeckung verpflichtete Fürsorgeleistung auf untere Lohngruppen entwickeln könnte. ${ }^{231}$ Im Beiratsausschuß sowie im DST hingegen überwog, auch auf sozialdemokratischer Seite, die Furcht vor einer nicht mehr bedarfsdeckenden Fürsorgeleistung. ${ }^{232}$ Angesichts dieser breiten Ablehnung arrangierte sich Muthesius gegenüber Scheffler und Gottschick Ende 1956 schließlich mit der Beibehaltung der Richtsätze. ${ }^{233}$ Nichtsdestoweniger artikulierte Muthesius vor dem Deutschen Blindenverband Anfang Juli 1957 seine Reformforderungen nochmals deutlicher denn je: Die Sicherung des notwendigen Lebensunterhalts sei eine soziale Verpflichtung der Allgemeinheit und gegenüber den fürsorgerischen Individualhilfen ein Fremdkörper; ohne allzu starkes Eindringen in die persönlichen Verhältnisse müsse sie auf „einfache, schlichte, der Würde der Persönlichkeit mehr gerecht werdende Weise“ auch örtlich durchgeführt werden. Da sie nicht von der Finanzkraft des örtlichen Trägers abhängig sein dürfe, müsse der Bund die Richtsätze festsetzen und diese Leistungen erheblich mitfinanzieren. ${ }^{234}$

Wenn die Vorschläge von Muthesius hier nochmals so ausführlich dargelegt wurden, dann weil der DV-Vorsitzende nach Kenntnis der Verfasserin der einzige war, der zu dieser Zeit noch ein echtes Alternativ-Konzept für einen - je nach Konjunkturverlauf: den - zentralen Bereich öffentlicher Fürsorgeleistung vorlegte. Sie blieben freilich Konzept. Die Gründe für die Ablehnung waren sehr viel mannigfaltiger, als es die Standardargumente von der notwendigen Individualisierung auch bei laufenden Leistungen, deren Verzahnung mit anderen Fürsorgeleistungen oder vom Kampf gegen den Versorgungsstaat ${ }^{235}$ zu erkennen ge-

230 Weitgehend zustimmend der DLT-Sozialausschuß zunächst am 5./6.9.1956, ablehnend dann am 8. 2.1957, vgl. Niederschriften, BAK, B 172/444-01/1.

231 Vgl. Besprechung mit den Länderfürsorgereferenten am 12.12.1956, BAK, B 106/ 9789/2, sowie die Niederschrift über die Sitzung des DLT-Sozialausschusses am 8.2. 1957, BAK, B 172/444-01/1.

232 Vgl. die Niederschriften über die Sitzung des Arbeitsausschusses für Fragen der Fürsorge am 1./2.2.1957, ADW, HGSt 6769, und die Sitzung des DST-Sozialausschusses am 6./7.6.1957 nebst Anlage, LAB, B Rep. 142-9, 1236.

233 Vgl. Vermerk Referat V A 4 vom 21.12.1956, BAK, B 106/20652.

234 Vgl. Heisig, Armenpolitik, 1995, S. 143; Die Blindenwelt 1958, H. 9, S. 1f.; ferner Giese, 25 Jahre, S.255, Anm. 12. Unterstützt wurde Muthesius auf dem Fürsorgetag 1957 nur von dem Stuttgarter Sozialamtsleiter Felix Mayer, Träger, S. 404ff.

$235 \mathrm{Vgl}$., auch für das Folgende, die Niederschriften über Sitzungen des Studienkreises „Soziale Neuordnung" am 25./26.3.1955, ADW, ZB 856, des Arbeitsausschusses für Fragen der Fürsorge am 21.1. und 6./7.4.1956 sowie am 1./2.2. und 14./15.6.1957, ADW, HGSt 6769, und des DLT-Sozialausschusses am 24.3.1955, BAK, B 172/444-01/1; ferner Schmerbeck, Bezirksfürsorgeverbände, S. 322; Schräder, Neuordnung, S. 342f.; auch Giese, 25 Jahre, S. 313f. 
ben: Ein wichtiger Grund war die verbreitete und nur von wenigen Experten nicht geteilte Annahme von der unaufhaltsamen Marginalisierung der laufenden Unterstützungen, die deren grundlegende Reform und die Begründung einer weiteren sozialen Sicherungsinstitution kaum zu rechtfertigen schien. ${ }^{236}$ Neben Hemnissen „vor allem verfassungs- und verwaltungsrechtlicher Art“237, die unmittelbare Bundeszuschüsse an die Gemeinden problematisch machten, hätte für die (gegenüber Zentralisierungstendenzen ohnehin empfindlichen) Länder eine Übertragung der laufenden Hilfen auf den Bund den teilweisen Verlust originärer, wenn auch der konkurrierenden Gesetzgebung unterliegender Zuständigkeit für die öffentliche Fürsorge (Art.74 GG) bedeutet. Aus kommunaler Sicht kam zu dem Verlust an Kompetenzen und Richtsatzspielräumen hinzu, daß seit der Pauschalierung der Kriegsfolgenhilfe ab 1. April 1955 über die Länder den Kommunen jährlich ohne Einzelnachweis feste Finanzzuweisungen des Bundes vor allem für die laufende Unterstützung für Kriegsfolgenhilfeempfänger zuflossen. Eine gesonderte staatliche Einkommenshilfe hätte diese Bundeszuweisungen weitgehend obsolet gemacht und damit die Kommunen einer wichtigen Einnahmequelle beraubt. Außerdem hätte eine unmittelbar bundesfinanzierte Hilfe die kommunale Verhandlungsposition bei der Reform der Finanzverfassung geschwächt, bei der die Kommunen gerade auch ihre hohen Belastungen im sozialen Bereich anführten. ${ }^{238}$ Aus Sicht des Bundesfinanzministeriums wiederum war weder eine Lockerung der Bedarfsprüfung mit unabsehbaren finanziellen Konsequenzen noch eine Trennung von Aufgaben- und Ausgabenverantwortung bei der Verwaltung von Bundesmitteln durch örtliche Stellen sonderlich attraktiv. Nicht zuletzt kam hinzu, daß innerhalb der regierenden CDU/CSU nach der Bundestagswahl 1957 versorgungsähnliche Modelle rapide an Prestige verloren.

So sprachen sich schließlich auch der Sozialausschuß des DST und der Fürsorgeausschuß des Beirats im Dezember 1957 endgültig dafür aus, die laufenden Unterstützungen bei der öffentlichen Fürsorge zu belassen, geregelt in einem besonderen Abschnitt des künftigen Bundesfürsorgegesetzes, beruhend auf den Prinzipien der Individualisierung und Nachrangigkeit, versehen mit einem Rechtsanspruch, orientiert am tatsächlichen, anhand von Richtsätzen festzustellenden Bedarf. 239 Das Scheitern des Muthesiusschen Modells einer gesonderten staatlichen Einkommenshilfe ist damit ein treffender Beleg für die „Pfadabhän-

236 Heinz Keese rechnete auch für die Zukunft mit einem „festen Block“ von ca. 1 Mio. HLU-Empfängern, hielt aber die Zeit für eine gesonderte staatliche Einkommenshilfe noch nicht für reif; vgl. dessen Referat vor der Gruppe IV des Studienkreises „Soziale Neuordnung“ am 16.7.1957, Anlage zur Niederschrift, LAB, B Rep. 142-9, 1263; ähnlich Weinbrenner, Grundfragen, S. 147f.; Weller, Hilfe, S.7.

237 Muthesius, Fürsorgeprinzip, S. 251; vgl. auch Mayer, Träger, S. 406.

238 Vgl. Entschließung des DLT-Sozialausschusses vom 8.11.1957, Anlage zur Niederschrift, BAK, B 172/444-01/1.

239 Vgl. Niederschrift über die Sitzung des DST-Sozialausschusses am 13./14.12.1957, LAB, B Rep. 142-9, 1282, und die offiziellen DST-Empfehlungen vom 1.3.1958, ebenda, 1283; Niederschrift über die Sitzung des Arbeitsausschusses für Fragen der Fürsorge am 4.12.1957, mit Anlage 1, ADW, HGSt 6769. 
gigkeit" auch der Fürsorgereform, die zwar soziale Leistungen durchaus ändern sollte, deren Trägerstrukturen und Finanzierungsmodi aber nicht ${ }^{240}$. Scheffler selbst erklärte auf dem Fürsorgetag 1957, es sei eben „nach der ganzen Entwicklung unserer sozialen Leistungen doch nicht richtig, für die Leistungen für den laufenden Lebensunterhalt ein Hilfssystem außerhalb der Institution der Fürsorge hinzustellen". .241

Tatsächlich legte die Sozialabteilung mit dem Referentenentwurf vom Juli 1958 eine Regelung der Hilfe zum Lebensunterhalt vor, die „im wesentlichen in einer zum Teil straffenden, zum Teil erweiternden Zusammenfassung des geltenden Rechtes" bestand. 242 Ähnlich wie $\ 5$ RGr. bestimmte der Entwurf, daß diese Hilfe nur erhalte, „wer für sich und seine unterhaltsberechtigten Angehörigen den notwendigen Lebensunterhalt nicht durch Einsatz seiner Arbeitskraft, seines Einkommens und seines Vermögens beschaffen kann". ${ }^{243}$ Anders als bei der Hilfe in besonderen Lebenslagen war also auch weiterhin grundsätzlich der volle Einsatz der eigenen Mittel erforderlich. ${ }^{244}$ Konkreter und großzügiger als bisher allerdings definierte der Entwurf in $\mathbb{1 4}$ das, was zum notwendigen Lebensunterhalt gehörte: „Ernährung, Unterkunft, Hausrat, Heizung, Kleidung, Körperpflege, den für die Führung des Haushalts erforderlichen Bedarf sowie sonstige persönliche Bedürfnisse des täglichen Lebens" ${ }^{245}$ Die Hilfe für diese besonderen Bedürfnisse sollte „dem Hilfeempfänger insbesondere Beziehungen zur Umwelt und die Teilnahme am kulturellen Leben ermöglichen "246 und war daher nun als Pflichtleistung auch für Anstaltsbewohner in Form eines Taschengeldes zu gewähren. Jetzt sollte also auch der Empfänger von Hilfe zum Lebensunterhalt über das bloße wirtschaftliche Existenzminimum hinaus einen „kulturellen Bedarf“ geltend

240 Vgl. Conrad, Alterssicherung, S.103f. Auch die Fürsorge stützt also die These, daß zumindest in den 1950er Jahren die bundesdeutsche Sozialstaatlichkeit sich als weitgehend resistent gegenüber dem für viele andere Bereiche typischen Trend der „westernization“ erwies; vgl. Hockerts Einführung, S. 10.

241 Gerhard Scheffler, Neuordnung, S. 23.

242 Gottschick, Referentenentwurf, S. 17.

243 \ 13 Abs. 1 des BSHG-Entwurfs 7/1958, BAK, B 106/20643.

244 Die Vorschriften über das einzusetzende Einkommen/Vermögen entsprachen im wesentlichen denjenigen des FÄG: Bei der Prüfung des Bedarfs durfte nur das Netto-Einkommen angesetzt werden und waren bestimmte Vermögenswerte (zur Sicherung der Lebensgrundlage, „kleines Hausgrundstück“, Erbstücke etc.), zweckbestimmte Leistungen (Pflegegeld etc.) und Zuwendungen der freien Wohlfahrtspflege oder freiwillige Leistungen ehemaliger Arbeitgeber weitgehend geschützt; um die Berechnung stärker zu vereinheitlichen, wurde jetzt die Bundesregierung zum Erlaß einer entsprechenden Rechtsverordnung ermächtigt; vgl. $\mathbb{S}$ 76-78, $\mathbb{S} 84 \mathrm{f}$. ebenda. Darüber hinaus sah $\mathbb{\$} 87$ wie bisher die Möglichkeit eines Darlehens vor.

245 So auch der Arbeitsausschuß für Fragen der Fürsorge am 4.12.1957; vgl. Niederschrift, Anlage 1, ADW, HGSt 6769. \$ 6 RGr. zählte zum Lebensunterhalt „insbesondere Unterkunft, Nahrung, Kleidung und Pflege“; die Hilfe zur Pflege gliederte der BSHGEntwurf als eigene HBL aus. Darüber hinaus war im Rahmen der HLU wie bisher die Zahlung der Bestattungskosten ( $\$ 16$ des Entwurfs) und erstmals (ohne Rechtsanspruch) „in angemessenem Umfang“ die Zahlung von Beiträgen für eine Altersvorsorge (\$15) möglich.

246 Scheffler dachte hier etwa an Kosten für Fahrgeld, Theater- oder Kinobesuch; vgl. Vermerk über Besprechung mit Abteilungsleiter am 7.2.1956, BAK, B 106/9688. 
machen können. ${ }^{247}$ Dieses im Zusammenhang mit dem „Warenkorb“ von 1955 schon diskutierte, mit den Verwaltungsvorschriften noch zaghaft avisierte ${ }^{248}$ Ziel war die Konsequenz des ja für alle Hilfeempfänger geltenden Auftrags, ein „den Anschauungen der Gemeinschaft entsprechendes Leben“ zu ermöglichen; gleichzeitig legitimierte diese stärkere Anpassung an die „gewandelten sozialen Anschauungen“ den Verbleib der laufenden Unterstützungen im Fürsorgesystem.

Daß eine gewisse Einschränkung des kommunalen Ermessens auch für die laufenden Unterstützungen von der Sozialabteilung durchaus beabsichtigt war, machte der Entwurf bei dem dafür entscheidenden Hebelpunkt deutlich: dem Richtsatz. Zwar unterschied der Entwurf wie bisher laufende und einmalige Leistungen, verhinderte also auch künftig die Einbeziehung der damit bisher abgedeckten Bedarfsgruppen (Heizung, Kleidung etc.) in den Richtsatz, was dessen unmittelbare Erhöhung und eine größere Dispositionsmöglichkeit des Hilfeempfängers bedeutet hätte, eine Linie, die vor allem der Hannoveraner Sozialamtsleiter Heinz Keese (SPD) verfocht. ${ }^{249}$ Immerhin aber erhob der Entwurf die einmaligen Hilfen jetzt zu Pflichtleistungen $(\mathbb{S} 21)$. Die laufenden Leistungen waren, wie es in geänderter Terminologie hieß, „nach Regelsätzen zu gewähren“, von denen nur abgewichen werden durfte, „wenn ein erhöhter Bedarf besteht“ (\ 22). ${ }^{250}$ Damit dies nicht zu einer Minimierung des Unterstützungsstandards führte, be-

247 Vgl. Niederschrift über die Sitzung des Arbeitsausschusses für Fragen der Fürsorge am 1./2.2.1957, ADW, HGSt 6769; ähnlich der DST-Arbeitskreis „Fürsorgerecht“ am 7./8.5.1957, Niederschrift, LAB, B Rep. 142-9, 1282.

248 Die VV über den Aufbau der Fürsorgerichtsätze vom 23.12.1955, GMBl. 1956 S. 58, schrieben u.a. die Einbeziehung „kleinerer Bedürfnisse verschiedener Art“ (Ziff. 2.h) vor.

249 Vgl. Keeses Referat vor der Gruppe IV des Studienkreises „Soziale Neuordnung“ am 16.7.1957, Ms., LAB, B Rep. 142-9, 1263; Keese, Richtsatzreform. Die Gewährung von einmaligen Beihilfen war in der Praxis zwar ebenfalls teilweise schematisiert, wurde aber nach wie vor an eine fürsorgerische Bedürftigkeitsprüfung geknüpft und daher als besonders diskriminierend empfunden; vgl. Ewald Wientgen im DLT-Sozialausschuß am 4.2.1955, Niederschrift, BAK, B 172/444-01/1. Die Einberechnung in den Richtsatz lehnte die Mehrheit im Richtsatz-Arbeitskreis ebenso wie der Fürsorgeausschuß des Beirats, der DLT und ein Teil der Länderreferenten vor allem mit der Begründung ab, daß erfahrungsgemäß die meisten Empfänger nicht ausreichend wirtschaften könnten, um für diese besonderen Bedürfnisse aus der laufenden Hilfe Rücklagen zu bilden; vgl. Besprechung mit den Fürsorgereferenten der Länder am 12.12.1956, BAK, B 106/9789/2; Vermerk Referat V A 4 vom 21.12.1956, BAK, B 106/20652; Niederschriften über die Sitzungen des Arbeitsausschusses für Fragen der Fürsorge am 1./2.2. und 14./15. 6.1957, ADW, HGSt 6769, der Gruppe IV des DV-Studienkreises am 16. 7.1957, ADW, HGSt, SP-S XXIIIc I/O, sowie des Arbeitskreises „Aufbau der Richtsätze“ am 3.10.1957, ADW, HGSt 7025; Schmerbeck, Bezirksfürsorgeverbände, S. 322, 324. Der Entwurf - wie auch das spätere BSHG - ließen allerdings offen, welcher Bedarf durch einmalige Leistungen zu decken sei; zu dieser Unklarheit im BSHG vgl. Giese, Regelsatzsystem, S. 513.

250 Der Wechsel der Terminologie ist von den Zeitgenossen und auch in der Gesetzesbegründung zumeist nur als formale Änderung im Kontext allgemein erneuerter Begrifflichkeit gedeutet worden; einzig Kurt Wehlitz verwies bereits bald nach Verabschiedung des BSHG auf die damit verbundene qualitative Verbesserung der Position des Hilfeempfängers, da jetzt die gesetzliche Bedarfsvermutung in der Regel detaillierte Bedürftigkeitsprüfungen ausschloß; vgl. Giese, Regelsatzsystem, S. 511f. 
stimmte der Entwurf, die Regelsätze müßten „eine den Anschauungen der Gemeinschaft entsprechende Lebensführung ermöglichen und den tatsächlichen Lebenshaltungskosten unter Berücksichtigung örtlicher Unterschiede entsprechen“, ein „Entbehrungsfaktor" war damit künftig ausgeschlossen.251 Die in den Richtsatz-Vorschriften von 1955 angelegte Abschaffung der berüchtigten „Auffanggrenze" sollte nun auch durch förmliches Gesetz erfolgen.

Festzusetzen waren die Regelsätze durch die jeweilige oberste Landesbehörde deren Definition durch die örtlichen Fürsorgeträger, wie es sie nur noch in Rheinland-Pfalz gab, war also nicht mehr vorgesehen, allerdings auch nicht eine politisch kaum durchsetzbare Festsetzung durch den Bund. Im Interesse einer stärkeren Vereinheitlichung verpflichtete allerdings $\$ 22$ den Bundesinnenminister, „mit Zustimmung des Bundesrates Verwaltungsvorschriften über den Aufbau der Regelsätze sowie über die Grundsätze für die Bemessung von Leistungen außerhalb der Regelsätze zu erlassen“. 252

Mindestsätze für einen Regelbedarf, neue Verwaltungsvorschriften ohne Beteiligung des Finanzministers, keine Regelsatzfestsetzung durch die örtlichen Träger, nicht zuletzt die Verpflichtung auf einen gesellschaftlicher Konvention entsprechenden Lebensstandard - diese Bestimmungen, die während der Vorarbeiten unter Scheffler zunehmend verschärft worden waren ${ }^{253}$, machten deutlich, daß man in der Sozialabteilung aus den Schwierigkeiten des ersten Warenkorbs gelernt hatte und gewillt war, stärker als bisher ein vertretbares soziales Mindestniveau der laufenden Hilfen im Rahmen des überkommenen Systems zu sichern. Das widersprach vor allem dem massiven Interesse der Landkreise, lag aber durchaus auf der Linie der einschlägigen Arbeitskreise des CDU-Parteitags Ende April 1956, die gefordert hatten, daß für „die Sicherstellung der Barunterstützungen zur Bestreitung des Lebensunterhaltes [...] vom Bund aus einheitliche Bestimmungen zu erlassen" seien. ${ }^{254}$ Soweit allerdings, wie von Muthesius und niedersächsischen Fürsorgereformern gewünscht, die konkrete Zusammensetzung der Richtsätze im Gesetz zu fixieren, wollten Gottschick und sein Abtei-

251 Pluskat, Gedanken, S. 308.

252 Marx und Muthesius hätten auch die Festsetzung der Richtsätze selbst lieber dem Bund übertragen, doch diesen massiven Eingriff in die Länderkompetenz hielt man im BMI realistischerweise für nicht durchsetzbar; vgl. Vermerk Referat V A 4 vom 21.12.1956, BAK, B 106/29652. Um dennoch eine zentrale, aber formal von der Bundesexekutive unabhängige Einflußnahme auf die Richtsätze zu ermöglichen, forderten Auerbach, Muthesius und Marx analog zur Rentenreform die Bildung eines „Sozialbeirats“ zur laufenden Überprüfung der Richtsätze und „Anpassung an die wirtschaftliche Entwicklung“, blieben aber ohne Resonanz; vgl. ebenda; ferner Niederschrift über die Sitzung des Arbeitsausschusses für Fragen der Fürsorge am 1./2.2.1957, ADW, HGSt 6769.

253 Die Bestimmung der Richtsätze als nicht mehr zu unterschreitende Regelsätze geschah wohl auf Anregung der Länderreferenten und von Marx und Muthesius; vgl. Besprechung mit den Referenten der Länder am 12.12.1956, BAK, B 106/9789/2; Vermerk Referat V A 4 vom 21.12.1956, BAK, B 106/20652; eine undatierte Version des $\mathbb{2 0}$ im BSHG-Entwurf vom 24.11.1956, BAK, B 106/20648, die offensichtlich einer Besprechung von Gottschick und Scheffler am 24.4.1957 zugrunde lag; vgl. Vermerk Referat V A 4 vom 24.4.1957, BAK, B 106/9789/2.

254 Lünendonk, CDU-Parteitag, S. 130. 
lungsleiter nicht gehen ${ }^{255}$, ebensowenig entschied der Entwurf die Frage der Bezugsgröße für die geforderte „den Anschauungen der Gemeinschaft entsprechende Lebensführung“ und damit deren mögliche Dynamisierung und laufende Überprüfung. Diese zentralen Probleme wurden vielmehr auf die künftigen Verwaltungsvorschriften delegiert und damit auch weiterhin außerhalb des parlamentarischen Kräftefeldes verhandelt. ${ }^{256}$ Bei allem, in erster Linie bei Scheffler angedeutetem Interesse, die Fürsorgeträger bei den laufenden Hilfen künftig etwas stärker an die Kandare modernisierter Sozialstandards zu nehmen, blieb die Sozialabteilung damit doch auf dem vertrauten richsatzpolitischen „Pfad“.

Auch bei der Frage des Mehrbedarfs folgte der erste Referentenentwurf den durch das FÄG seinerzeit vorgezeichneten Wegen, verschärfte aber teilweise die Regelungen zugunsten der Hilfeempfänger und erweiterte den Kreis der Berechtigten auf alle Behinderten sowie werdende Mütter. ${ }^{257}$ Schefflers Bemühungen, über den Mehrbedarf hinaus typische Tatbestände zu erfassen, waren allerdings auf wenig positive Resonanz gestoßen und fanden keinen Eingang in den ersten Referentenentwurf. Scheffler hatte seit Anfang 1956 wiederholt angeregt, bei „Personen, die besonders auf die Hilfe der Allgemeinheit angewiesen seien, z.B. Halbfamilien, Alte“ möglicherweise „im Rahmen einer Gruppenbildung von einer finalen Betrachtung zu einer kausalen“ zurückzukehren. ${ }^{258}$ Pendant dieser positiven sollte eine negative Typisierung sein in Form einer „Absetzung der

255 Vgl. Niederschrift der Sitzung der Gruppe IV des DV-Studienkreises, 16.7.1957, ADW, HGSt, SP-S XXIIIc I/O.

256 Damit entsprach der Entwurf dem Diskussionsstand des mittlerweile reaktivierten DVArbeitskreises „Aufbau der Richtsätze“: Die vielfach ungenügende Anpassung der Richtsätze an die aktuelle Preisentwicklung nahmen die nördlichen Bundesländer Ende 1956 zum Anlaß, den ersten Warenkorb überprüfen zu lassen. Entscheidend war - auch im Vorfeld der Bundestagswahlen - die Frage, inwieweit nach den Sozialrentnern auch die Fürsorgeempfänger stärker an der allgemeinen Wohlstandsentwicklung teilhaben könnten. Während vor allem Fürsorgeexperten aus dem sozialdemokratisch regierten Niedersachsen eine möglichst weitgehende Pauschalierung forderten (Einbeziehung der Mehrbedarfszuschläge und der bisherigen einmaligen Leistungen in die Richtsätze), empfahl der Arbeitskreis Anfang 1958 nur zurückhaltende Korrekturen des bestehenden Warenkorbes und hielt an nach oben und unten elastischen Richtsätzen fest; vgl. Heisig, Armenpolitik, 1995, S. 167ff.; BldW 105 (1958), S. 137.

257 Vgl. SS 23-29 des BSHG-Entwurfs 7/1958, BAK, B 106/20643. Für alte Menschen, Behinderte und Schwangere war jetzt ein Mehrbedarf von mindestens 20\% verbindlich vorgeschrieben sowie ein nicht spezifizierter Mehrbedarf für Kinder und Jugendliche, um die bei den Richtsätzen für Minderjährige oft knauserigen Fürsorgeträger stärker in die Pflicht zu nehmen. Da der Teufel wie so oft im Detail steckte, versuchte $\$ 29$ außerdem zum Vorteil des Hilfeempfängers klarzustellen, daß bei Zusammentreffen der verschiedenen Voraussetzungen die Mehrbedarfe zu kumulieren waren - eine Frage, die in der Vergangenheit häufig zu Gerichtsverfahren Anlaß gegeben hatte; vgl. Jehle, Fürsorgerecht, S. 185ff. Daß dieser Versuch noch nicht ganz geglückt war, belegt die Tatsache, daß $\ 29$ theoretisch den kumulierten Mehrbedarf für eine über 65jährige werdende Mutter vorsah.

258 Niederschriften über die Sitzungen des Arbeitsausschusses für Fragen der Fürsorge am 21.2. und 6./7.4.1956, ADW, HGSt 6769; vgl. ferner Vermerk über Besprechung mit Abteilungsleiter am 7.2.1956, BAK, B 106/9688; Anlage zu Scheffler an Heusler etc., 9.2.1956, BAK, B 106/9789/2. 
Massnahmen für asoziale Personen von den sonstigen Hilfsmassnahmen durch das Gesetz“. ${ }^{259}$ Damit griff Scheffler ein altes Anliegen der Sozialabteilung auf: das sich durch „die Nachbarschaft zu den Asozialen ergebende Odium auszuräumen“. ${ }^{260}$ Wie seinerzeit die Mehrheit des DV-Studienkreises machte auch der Beirats-Fürsorgeausschuß gegen eine derartige legislatorische und verwaltungsmäßige Sonderbehandlung der zahlenmäßig unbedeutenden Gruppe der „ständigen Kunden" keine prinzipiellen, sondern nur praktische Gründe geltend, zumal, so der Frankfurter Stadtrat Prestel, es ,in der Fürsorge naturgemäss stets viele Menschen [gebe], bei denen Schuld und Schicksal nicht klar zu trennen sind, weshalb eine endgültige Beseitigung des Odiums nicht erwartet werden könne“. 261

Die Stellungnahmen der Fürsorgeträger zur Regelung der laufenden Unterstützungen im ersten Referentenentwurf richteten sich vor allem gegen die geplanten Bestimmungen zum Regelsatz und die vergleichsweise großzügige Auffassung des kulturellen Existenzminimums. Während der DST keinen Anstoß an der Verpflichtung zu Mindestregelsätzen nahm ${ }^{262}$, forderten die Mehrzahl der Länderreferenten wie auch der DLT, der DGT und der reaktivierte DV-Arbeitskreis „Aufbau der Richtsätze“ einmütig, zur Berücksichtigung örtlicher Unterschiede weiterhin die Regelsätze zumindest im Einzelfall auch unterschreiten zu können. ${ }^{263}$ Ebenso plädierten sämtliche kommunalen Spitzenverbände und die Mehrheit der Ländervertreter dafür, auch künftig eine Festsetzung der Regelsätze durch die kommunalen Träger vorzusehen. Tatsächlich gaben Duntze und Gottschick beiden Forderungen statt. Ebenso verzichtete der zweite Referentenentwurf, wie in den meisten dieser Stellungnahmen gewünscht, auf die abermalige ausdrückliche Verpflichtung auf ein „menschenwürdiges Leben“. ${ }^{264}$ Und nicht zuletzt war für die geplante Regelsatz-Verordnung vorgesehen, daß diese nun auch „das Verhältnis der Regelsätze zum Arbeitseinkommen“ zu regeln habe ${ }^{265}$ -

259 Niederschrift über die Sitzung des Arbeitsausschusses am 21.2.1956, ADW, HGSt 6769.

260 Scheffler auf der Konferenz der Wohlfahrtsminister am 29. 5. 1956, Kurzfassung des Referats, BAK, B 106/20652. Kitz, Fürsorgeprinzip, S.523, hatte es für ein zentrales Ziel der Fürsorgereform erklärt, im Aufbau des Gesetzes und in der Praxis der Ämter eine "Zwischenwand“ einzuziehen zwischen Personen, „die schuldlos in ihre Notlage hineingekommen sind“, und solchen, „die saft- und kraftlos sind, keinen Willen und keine Fähigkeit zur Eigenhilfe haben, aus zerrütteten Familienverhältnissen stammen oder sonst irgendeinen [...] , Makel' an sich haben".

261 Niederschrift über die Sitzung des Arbeitsausschusses für Fragen der Fürsorge am 6./7.4.1956, ADW, HGSt 6769.

262 Vgl. DST-Stellungnahme vom 27.11.1958, BAK, B 106/9686.

263 Vgl., auch zum Folgenden, Niederschrift über die Besprechung mit Vertretern der obersten Landessozialbehörden am 21./22. 10.1958, LAS Abt. 761 Nr. 8874; Äußerungen aus den Fachgremien des DV [5.11.1958], zu $\ 22$, sowie die Stellungnahmen des DLT vom 17.11.1958 und des DGT vom 1.12.1958, BAK, B 106/9686.

264 Der DST fürchtete, daß dadurch die Höhe der Regelsätze in das Benehmen der Verwaltungsgerichte gestellt werden könnte; DST-Stellungnahme vom 27.11.1958, BAK, B 106/9686.

265 Vgl. $\ 19$ Abs. 2 des BSHG-Entwurfs 3/1959, BAK, B 106/20646. 
damit, so der SPD-Fürsorgeexperte Willy Könen später, könne nun doch „die berühmte Auffanggrenze wieder fröhlich Urständ feiern“. 266

Die Gründe für dieses weitreichende Einlenken lassen sich aufgrund der Quellenlage nur vermuten: Möglicherweise erachteten die Ministerialbeamten angesichts des Widerstands der meisten Länderreferenten eine Zustimmung des Bundesrates zu der bisher vorgesehenen Regelung ohnehin für unwahrscheinlich. Außerdem hatte Gottschick schon früher wegen der noch erheblichen Meinungsverschiedenheiten weitergehende Vorschriften zu den Regelsätzen skeptisch beurteilt $^{267}$; möglicherweise teilte auch Duntze diese Vorbehalte stärker als sein Vorgänger. Nicht auszuschließen ist auch, daß man diese Lockerungen in der Sozialabteilung auch gar nicht mehr für so entscheidend angesichts einer anderen Änderung hielt: Auf Wunsch des Arbeitsministeriums schrieb der zweite Referentenentwurf anstelle von Verwaltungsvorschriften eine Rechtsverordnung über die Regelsätze vor, die im Einvernehmen mit den Bundesministern für Arbeit und Finanzen mit Zustimmung des Bundesrates zu erlassen war. ${ }^{268}$ Ungeachtet des aus ihrer Sicht vorteilhaften Mitspracherechts des Finanzministers würde eine solche Verordnung die Fürsorgeträger ungleich stärker an die Bundesvorgaben binden als die bisherigen Verwaltungsvorschriften und ein vielgefürchtetes Mitspracherecht der Verwaltungsgerichte begründen können. ${ }^{269}$

Auch bei der Frage des avisierten Lebensstandards lenkte die Sozialabteilung ein: Zwar wurde nicht, wie von den Fürsorgeträgern und einigen Ländern gefordert, die Einbeziehung kultureller und sozialer Bedürfnisse überhaupt gestrichen, aber durch den Zusatz „in vertretbarem Umfang“ entschärft. ${ }^{270}$ Insgesamt also war den Bestimmungen über die laufenden Unterhaltsleistungen an entscheidenden Stellen der Stachel genommen. Anfang Juli 1959 stellte der DLT-Sozialausschuß daher befriedigt fest, die Regelsatz-Vorschriften seien „weitgehend entsprechend unseren Forderungen geändert worden“.271

In der zweiten Überarbeitungsphase erlebten diese Vorschriften bis auf eine Ausnahme keine gravierenden Änderungen mehr: Auf Wunsch verschiedener Länder, vor allem aber auf Druck des durch Elsholz vertretenen Finanzministeriums verzichtete das Innenministerium schließlich darauf, den Mehrbedarf als

266 BT, 3. Wp. 1957, Sten. Ber., Bd. 45, S. 6260.

267 Sitzungsniederschrift der Gruppe IV des DV-Studienkreises am 16.7.1957, ADW, HGSt, SP-S XXIIIc I/o.

268 Vgl. Vermerk des Referats V 4 vom 23.10.1958, BAK, B 106/9686. Diese Verordnung betraf Inhalt und Aufbau der Regelsätze selbst, ferner laufende Leistungen außerhalb der Regelsätze, vor allem Mieten, sowie nun wieder das Verhältnis der Regelsätze zum Arbeitseinkommen; vgl. $\ 19$ des BSHG-Entwurfs 3/1959, BAK, B 106/20646.

$269 \mathrm{Da}$ sie durch Anordnung von Organen der vollziehenden Gewalt und nicht im förmlichen Gesetzgebungsverfahren ergeht, ist die Rechtsverordnung zwar nicht im formalen, wohl aber im materiellen Sinn Gesetz mit allgemeinverbindlichem Charakter. Verwaltungsvorschriften hingegen besitzen nur verwaltungsinterne Verbindlichkeit und begründen kein unmittelbares richterliches Prüfungsrecht.

270 Vgl. \11 des BSHG-Entwurfs 3/1959, BAK, B 106/20646.

271 Niederschrift über die Sitzung des DLT-Sozialausschusses am 2./3.7.1959, BAK, B 172/444-02/2, Beiheft. 
einen Mindestmehrbedarf $\mathrm{zu}$ definieren. ${ }^{272}$ Das im ersten Referentenentwurf deutliche Bemühen, bei der Gewährung der Hilfe zum Lebensunterhalt eine stärkere Standardisierung und Verpflichtung auf ein kulturelles Mindestniveau herbeizuführen, war damit im Regierungsentwurf wieder deutlich reduziert worden. Würde der Entwurf in dieser Form Gesetz, hing die künftige Minimalsicherung der staatsbürgerlichen Existenz entscheidend von der extra-parlamentarischen Ausgestaltung der Regelsatz-Verordnung und der Überarbeitung des Warenkorbs $\mathrm{ab}$.

\section{Hilfe zur Arbeit und Arbeitspflicht}

Mit dem Unterabschnitt „Hilfe zur Arbeit“ unternahm die Sozialabteilung den Versuch, die juristisch diffizile und inhaltlich schwierig zu umgrenzende Materie der Arbeitsfürsorge im Zusammenhang der Hilfe zum Lebensunterhalt neu zu ordnen. In enger Anlehnung an eigene Ideen der Weimarer Zeit hatte Theodor Marx 1952 „Arbeitsfürsorge“ definiert als das weite Feld „derjenigen Sondermaßnahmen zur Entwicklung, Pflege und Erhaltung der Arbeitskraft [...], die um der persönlichen Eigenart Einzelner oder in der Auswirkung wirtschaftlich-sozialer Zustände auf den Einzelnen notwendig sind, um hilfsbedürftige Menschen an das Durchschnittsmaß einer [...] durch sozialpolitische Einrichtungen gewährleisteten Existenzsicherung heranzubringen". ${ }^{273}$ Dieser Ansatz umfaßte die zunehmend als „Rehabilitation“ bezeichneten therapeutischen Hilfen für Erwerbsbeschränkte ebenso wie Arbeitsbeschaffungsmaßnahmen für Arbeitslose, Ausbildungshilfen für Jugendliche, psychologische und sozialpädagogische Eingliederungshilfen für "Gefährdete“ oder Arbeitstests zur Disziplinierung „Arbeitsscheuer“ und umschrieb damit keineswegs auf die öffentliche Fürsorge beschränkte Tätigkeitsfelder. Im Gegenteil: Gerade Zuständigkeitskonflikte mit der Arbeitsverwaltung beherrschten bis zur Reform der Arbeitslosenversicherung 1956/57 die Diskussionen über die Arbeitsfürsorge. ${ }^{274}$ In der Fürsorgereform selbst wurde ein wesentlicher Teil dieser Aufgaben jeweils gesondert im Rahmen der Hilfe in besonderen Lebenslagen neu geregelt.

Arbeitsfürsorge in dem hier interessierenden engeren, fürsorgerechtlichen Sinne meinte, daß nach $\mathbb{1} 19$ RFV Unterstützung für arbeitsfähige Hilfsbedürftige in Form von angemessener gemeinnütziger Arbeit gewährt (Fürsorgearbeit) oder aber überhaupt von der Leistung solcher Arbeit abhängig gemacht werden konnte (Pflichtarbeit). Daneben statuierte $\mathbb{7}$ RGr. eine Arbeitspflicht, die allerdings

272 Vgl. BMF am BMI, 2. 5.1959; Vermerk Referat V 4 vom 10.6.1959, BAK, B 106/20644; Änderungsvorschläge der Länder vom 28./29.4.1959, BAK, B 106/20647. In \21 des Regierungsentwurfs blieb nur der Mindestmehrbedarf für Blinde erhalten; auf Vorschlag Gottschicks wurde in den übrigen Fällen stattdessen die Kautel eingefügt, „soweit nicht im Einzelfall ein höherer Bedarf besteht“, BT, 3. Wp. 1957, Anlage, Bd. 67, Drs. 1799.

273 Marx, Auftrag, S. 372. Zur hier dominierenden Rolle von Marx innerhalb des DV und zu seinen arbeitsfürsorgerischen Aktivitäten als Stadtrat von Nürnberg vgl. NDV 38 (1958), S. 201ff.; ferner NDV 35 (1955), S. $75 f f$.

274 Vgl. die Fürsorgetage 1952, NDV 32 (1952), S.376ff., und 1955, Fürsorge und Sozialreform, S. $343 \mathrm{ff}$. 
durch Zumutbarkeitsklauseln gemildert wurde, und bestimmte, daß die Fürsorge dem Hilfsbedürftigen, „soweit möglich“, Gelegenheit zur Arbeit bieten solle. ${ }^{275}$

Rechtlich wie in der Anwendung unterschieden sich die beiden in der Praxis daraus entwickelten Typen gemeindlicher Arbeitsfürsorge: Bei der Pflichtarbeit überwog der Zwangscharakter, Fürsorgearbeit erforderte das Einverständnis des Unterstützten. Während der Pflichtarbeiter keinen Lohn, sondern richtsatzmäßige Unterstützung plus Aufwandsentschädigung erhielt, die zusammen nicht die Höhe des Tariflohnes erreichten, bekam der Fürsorgearbeiter Anfang der fünfziger Jahre meist ein Entgelt, das der tariflichen Bezahlung ungelernter Gemeindearbeiter entsprach. Anders als die Pflichtarbeit war Fürsorgearbeit meist versicherungspflichtig. ${ }^{276}$ Die rund vier Arbeitsstunden täglich umfassende Pflichtarbeit wurde „überwiegend zur Prüfung des Arbeitswillens und auch der Hilfsbedürftigkeit angewendet“, etwa um Schwarzarbeit auszuschließen; ein Nürnberger Fürsorgebeamter verglich sie 1957 mit einem „Sieb, das die echten Hilfsbedürftigen von den unechten scheidet“, und schätzte, daß die Abschreckung durch drohende Pflichtarbeit „beträchtlich[e]“ Einsparungen bringe. ${ }^{277}$ Demgegenüber galten die verschiedenen Formen der Fürsorgearbeit als besonders geeignet, um Arbeitswillige wieder in den normalen Arbeitsprozeß einzugliedern.

Obwohl theoretisch breiter Konsens über die Notwendigkeit kommunaler Arbeitsfürsorge als der „sozial würdigsten, finanziell günstigsten und ethisch wertvollsten Hilfe " 278 bestand, blieb deren quantitative Bedeutung auch in den fünfziger Jahren trotz zunächst beträchtlicher Arbeitslosigkeit relativ gering. ${ }^{279}$ Das galt vor allem für die Fürsorgearbeit, zumal in den Ländern der ehemaligen britischen Zone ohnehin der Anreiz für solche Aktivitäten der Kommunen fehlte, da fast alle Arbeitslosen hier vom Arbeitsamt unterstützt wurden. ${ }^{280} \mathrm{Zu}$ den ungeklärten $\mathrm{Zu}$ ständigkeiten von Arbeitsamt und Kommunen kamen die hohen Kosten für kommunale Arbeitseinrichtungen ${ }^{281}$ und rechtliche Unklarheiten, insbesondere wegen der erforderlichen „Gemeinnützigkeit“; fehlte deren wesentliches Element, die „Zusätzlichkeit“, bescheinigten die Gerichte den Unterstützten immer wieder, in einem regulären privatrechtlichen Arbeitsverhältnis mit allen tarifrechtlichen

$275 \mathrm{Ob}$ eine Arbeit „billigerweise“ zumutbar war, sollte „nach Lebensalter, Gesundheitszustand, häuslichen Verhältnissen und, soweit angängig, auch nach der beruflichen Ausbildung beurteilt werden“ ( $\mathbb{7}$ Abs. 2 RGr.). Bei Frauen war Erwerbsarbeit auch nicht zumutbar, „wenn dadurch die geordnete Erziehung ihrer Kinder gefährdet würde“, und Pflichten im Haushalt oder bei der Pflege von Angehörigen waren besonders zu berücksichtigen (Abs. 3).

276 Vgl. ausführlich Petersen, Arbeitsfürsorge, S. 407ff.; Kurth, Recht; Staudacher, Arbeitsfürsorge; Jehle, Fürsorgerecht, S. 42ff. Aufgrund von Bundesvorschriften von 1951/52 entfiel für beide Formen in der Regel die Rückerstattungspflicht.

277 Staudacher, Arbeitsfürsorge, S. 8.

278 Ebenda, S. 11.

279 Mangels konkreter Zahlen vgl. die Ergebnisse einer DV-Umfrage vom Sommer 1954, NDV 35 (1955), S. 288ff.; Marx, Die Städte zur Neuordnung, S. 60. Zur arbeitsfürsorgerischen Praxis in einzelnen Großstädten: Marx, Arbeitsfürsorge; Wöhrmann, Arbeitsfürsorge.

280 Vgl. NDV 35 (1955), S. 75; 37 (1957), S. 1.

281 Vgl. Marx, Aufgabenabgrenzung, S. 381. 
Konsequenzen zu stehen. ${ }^{282}$ Mit dem zunehmenden Arbeitskräftemangel seit Mitte der fünfziger Jahre stiegen jedoch die Chancen, auch bislang als nicht vermittlungsfähig geltende, von der Fürsorge betreute „halbe Kräfte“ im normalen Arbeitsmarkt unterzubringen, so daß die von vielen Fürsorgeexperten neben dem Ausbau der Rehabilitation geforderte Intensivierung der gemeindlichen Arbeitsfürsorge auch aus fiskalischer Perspektive sinnvoll erschien. ${ }^{283}$

Gerade hier wurde die für die fünfziger Jahre noch so typische Mischung von paternalistisch-disziplinierender, mit finanziellen Erwägungen gepaarter Wertmuster und sozial-integrativer, der individuellen Persönlichkeitsbildung verpflichteter Elemente besonders deutlich: Neben der Auffassung, daß fürsorgerische Hilfe zur Arbeit auch der „Entfaltung der Persönlichkeit“ diene ${ }^{284}$, stand die Meinung, die Arbeitsfürsorge habe den „unserer Hilfe bedürftigen Menschen [...] mit Einschaltung seines Willens soweit zu formen [...], daß er bestmöglich für die Allgemeinheit eingesetzt werden kann“".285 Danach waren die traditionellen Instrumentarien der Fürsorgearbeit und der Pflichtarbeit auch künftig beizubehalten, allerdings eine Akzentverschiebung zugunsten der Fürsorgearbeit und dementsprechend nur eine graduelle Reform des Fürsorgerechts notwendig. 286

Mit diesen Fragen befaßte sich vornehmlich ein im März 1953 auf Initiative von Marx gegründeter und später von ihm geleiteter DV-Fachausschuß aus Vertretern der öffentlichen Fürsorge und der Arbeitsverwaltung, an dessen Sitzungen häufig auch Beamte der Sozialabteilung teilnahmen. ${ }^{287}$ Von Marx in enger Fühlungnahme mit der Sozialabteilung formulierte Leitsätze bildeten so auch die Grundlage für die entsprechenden Empfehlungen des Fürsorgeausschusses des Beirats Ende Januar 1958: Die Fürsorgeträger müssten künftig dem Hilfesuchenden in enger Zusammenarbeit mit den Arbeitsämtern zu einem zumutbaren Arbeitsplatz verhelfen. War das nicht möglich, sollte arbeitsfähigen und arbeitswilligen Hilfesuchenden grundsätzlich statt Geldleistung gemeinnützige Fürsorgearbeit ermöglicht werden. In Umkehrung der bisherigen Praxis war Pflichtarbeit nur noch in den Ausnahmefällen von „Arbeitsunwilligkeit oder Arbeitsscheu“ einzusetzen. ${ }^{288}$

282 Vgl. Petersen, Arbeitsfürsorge, S. 408; Kurth, Arbeitsfürsorge, S. 318; ders., Recht, S. 201.

283 Vgl. NDV 37 (1957), S. $1 \mathrm{ff}$.

284 Staudacher, Arbeitsfürsorge, S.7. Der SPD-Sozialplan forderte einen „sozialpädagogischen Charakter" der Arbeitsfürsorge; vgl. Sozialplan, S. 127.

285 So der Hamburger Verwaltungsdirektor Bernhard Wöhrmann auf dem Fürsorgetag 1957, Neuordnung des Fürsorgerechts, S. 221. Dieser „arbeitserzieherische“ Ansatz zur Mobilisierung von Arbeitskräften war ganz ähnlich bereits auf dem Fürsorgetag 1938, also ebenfalls in einer Zeit des Arbeitskräftemangels, von Fürsorgepolitikern vertreten worden; vgl. Rudloff, Fürsorge, S. 201.

286 Vgl. etwa Kurth, Arbeitsfürsorge, S. 318.

287 Vgl. NDV 33 (1953), S. 131; 38 (1958), S. 203.

288 Bislang verpflichtete $\ 6$ RGr. die Fürsorgeträger nur zur „Wiederherstellung der Arbeitskraft", was vor allem medizinisch interpretiert wurde; vgl. Muthesius, Grundlagen, S. 87. Vgl. insgesamt Neuordnung des Fürsorgerechts, S. 180, 213ff., 432; Niederschriften über die Sitzungen des Ständigen Ausschusses für gemeinsame Fragen der Fürsorge und der Arbeitsverwaltung am 14.1.1958, mit Anlage, ADW, HGSt, SP-S XXIIIc I/O; des Arbeitsausschusses für Fragen der Fürsorge am 4.12.1957, mit Anlage 1, sowie am 30./31.1.1958, mit Anlage 7, ADW, HGSt 6769; NDV 38 (1958), S. 308; Kurth, Arbeitsfürsorge, S. $319 \mathrm{ff}$. 
Denn auch weiterhin sollte gegebenenfalls die Leistung von der Arbeitsbereitschaft abhängig gemacht werden, allerdings nur bei der Hilfe zum Lebensunterhalt.

Anders als von Marx und anderen Experten im Interesse größerer Rechtssicherheit gewünscht, definierten die Empfehlungen des Beirats-Fürsorgeausschusses nur die künftige Form der Pflichtarbeit genauer, bei der neuen Form der Fürsorgearbeit hingegen bestanden offensichtlich unüberbrückbare Gegensätze zwischen der gewerkschaftsnahen Linie und einer vor allem im DLT beheimateten Richtung: Bisheriger Praxis entsprechend hatte Marx die gesetzliche Fixierung eines versicherungspflichtigen Arbeitsverhältnisses mit tarifangeglichener Entlohnung befürwortet. Während Walter Henkelmann vom DGB befürchtete, daß dann per Gesetz eine „besondere, ungünstig gestellte Arbeitnehmergruppe geschaffen werde“289, ging eine solche Regelung anderen Ausschußmitgliedern zu weit. 290

Der Referentenentwurf vom Juli 1958 entsprach dann weitgehend der Beschlußlage im Beiratsausschuß und statuierte ausdrücklich die Pflicht des Fürsorgeträgers zur Hilfe bei der Beschaffung zumutbarer Arbeit bzw. zur Förderung der Arbeitsbereitschaft. ${ }^{291} \mathrm{Ob}$ und wie die Fürsorgeträger allerdings Arbeitsbeschaffungsmaßnahmen ergriffen, blieb weiterhin ihrem Ermessen überlassen; statt ein neues Rechtskonstrukt der Fürsorgearbeit zu begründen, sah der Entwurf neben der Schaffung regulärer auch die Schaffung gemeinnütziger und zusätzlicher Arbeitsgelegenheit vor, die entweder normal entlohnt oder aber, der Regelung der "Gemeinschaftsarbeit" in der Novelle zur Arbeitslosenversicherung entsprechend, mit Hilfe zum Lebensunterhalt und einer Aufwandsentschädigung abgegolten werden konnte; in letzterem Fall bestand keine Versicherungspflicht. ${ }^{292}$ Außerdem sollte wie bei der bisherigen Pflichtarbeit, aber ohne die schwierige Voraussetzung der Gemeinnützigkeit, dem Hilfesuchenden gegebenenfalls ,geeignete Arbeit zugewiesen werden“ (\$20), während der ebenfalls Hilfe zum Lebensunterhalt und Aufwandsentschädigung gezahlt und kein versicherungspflichtiges Arbeitsverhältnis begründet wurde.

Alles in allem war damit der erste Referentenentwurf nur sehr bedingt dazu geeignet, die Sozialhilfeträger stärker als bisher zu - vor allem regulären Arbeitsverhältnissen angenäherten - Arbeitsbeschaffungsmaßnahmen jenseits der Überprüfung von Arbeitswilligkeit zu animieren. Das wurde auch im DV-Fachausschuß bemängelt. ${ }^{293}$ Vor allem das Bundesarbeitsministerium drängte jedoch auf noch stärkere Koordination mit dem AVAVG, so daß die Schonvorschriften bei der Zumutbarkeit von Arbeit wieder gelockert und der Zwangscharakter des $\ 20 \mathrm{ab}$ -

289 Vgl. Niederschrift über die Sitzung des Arbeitsausschusses für Fragen der Fürsorge am 30./31.1.1958, ADW, HGSt 6769.

290 Vgl. die ablehnende Haltung des DLT-Sozialausschusses am 8.11.1957, Niederschrift, BAK, B 172/444-01/1. Vermutlich bestanden auch auf Seiten des BMA Vorbehalte gegen eine solche Sonderregelung, die ja u.U. in gemeinnütziger Arbeit stehende Sozialhilfeempfänger besser stellte als Bezieher von Arbeitslosenhilfe.

291 Vgl. $\int 18,3$ des BSHG-Entwurfs 7/1958, BAK, B 106/20643.

$292 \int 19$ ebenda; vgl. $\int 142$ AVAVG in der Fassung vom 3. 4. 1957, BGBl. I S. 322.

$293 \mathrm{Vgl}$. Stellungnahme aus den DV-Fachgremien [5.11.1958], zu \19, BAK, B 106/9686. 
gemildert wurde: Bereits der zweite Referentenentwurf sah zur Gewöhnung bzw. Prüfung vor, daß dem Hilfesuchenden eine "geeignete Tätigkeit angeboten werden" solle. ${ }^{294}$ Nachdem auf Vorschlag des Bundesrats der Schutz von Müttern wieder verstärkt worden war, wurden diese Bestimmungen schließlich $1961 \mathrm{Ge}-$ setz. ${ }^{295}$

Der mit dem BSHG avisierte Auftrieb der kommunalen Arbeitsfürsorge blieb zunächst aus. Erst seit der Arbeitsmarktkrise der siebziger Jahre gewann die „Hilfe zur Arbeit" an Bedeutung, freilich lange in Form der nicht-tariflichen gemeinnützigen Arbeit, die, etwa im Rahmen der Betreuung von Asylsuchenden, primär zum Test der Arbeitswilligkeit mit abschreckender Wirkung eingesetzt wurde. Bis Ende der achtziger Jahre verschoben sich zwar die Gewichte zugunsten der tariflichen Sozialhilfearbeit: Doch die Konsequenz war selten die Rückführung in den „ersten Arbeitsmarkt", fast immer aber die Rückverweisung an das Arbeitsamt und damit eine finanzielle Entlastung der Kommunen. ${ }^{296} \mathrm{Zu}$ der von den Fürsorgereformern der fünfziger Jahre erhofften, bei einem großen Arbeitslosenanteil unter den Sozialhilfeempfängern umso notwendiger erscheinenden aktiven Arbeitsbeschaffungspolitik der Kommunen ist es damit bis in jüngste Zeit nur in Ausnahmen gekommen. Inwieweit die aktuelle Zusammenlegung von Arbeitslosen- und Sozialhilfe hier zu neuen Lösungen führt, bleibt abzuwarten.

Wenn auch im neuen Sozialhilferecht mit der „Hilfe zur Arbeit“ als „beste und wirksamste Hilfe für den Lebensunterhalt “297 die Akzente zugunsten sozialisierender Arbeitsbeschaffungsmaßnahmen verschoben wurden, blieben doch genügend andere Möglichkeiten, mangelnde Arbeitswilligkeit und andere ebenfalls als "asozial“ konnotierte Verhaltensmuster wie „unwirtschaftliches Verhalten“ zu sanktionieren. Nach bisherigem Fürsorgerecht waren bei „Arbeitsscheu oder offenbar unwirtschaftlichem Verhalten“ die Fürsorgeleistungen „auf das zur Fristung des Lebens Unerläßliche zu beschränken“ oder die Betroffenen in eine Anstalt einzuweisen; negative Auswirkungen auf Angehörige oder Haushaltsangehörige waren, soweit möglich, zu verhindern ( $\mathbb{1 3}$ RGr.). Darüber hinaus konnte, „wer, obwohl arbeitsfähig, infolge eines sittlichen Verschuldens der öffentlichen Fürsorge selbst anheimfällt oder einen Unterhaltsberechtigten anheimfallen läßt", wenn er beharrlich Arbeit ablehnte oder sich der Unterhaltspflicht entzog, durch die Fürsorgebehörde in ein geschlossenes Arbeitshaus zwangsweise eingewiesen werden $(\$ 20 \mathrm{RFV}) .{ }^{298}$ Während in den zwanziger Jahren nur selten

294 Vgl. Vermerk des BMI-Referats V 4 vom 23.10.1958; BMW an BMI am 4.12.1958, ebenda; $\mathbb{S} 17$ des BSHG-Entwurfs 3/1959, BAK, B 106/20646; $\mathbb{1 6}$ Abs.3 des Regierungsentwurfs vom Februar 1960, BT, 3. Wp. 1957, Anlagen, Bd.67, Drs. 1799; ferner die auf der Ressortbesprechung am 6./8.5.1959 gemachten Änderungsvorschläge, BAK, B 106/20647.

295 Vgl. Stellungnahme des Bundesrats zu $\mathbb{1 6}$ des Regierungsentwurfs, S. 70, und Auffassung der Bundesregierung, S. 82, BT, 3. Wp. 1957, Anlagen, Bd.67, Drs. 1799; $\mathbb{S}$ 18-20 BSHG.

296 Vgl. Rudolph, Kooperation, S. 89f.; Rudloff, Fürsorge, S. $205 f$.

297 Gottschick, Referentenentwurf, S. 17.

298 "Sittliches Verschulden“ umfaßte nach zeitgenössischer wie auch späterer Auslegung „Arbeitsscheu“, „Trunksucht", „Spielsucht“, „Verschwendung“ oder „geschlechtliche 
zu diesen Zwangsmaßnahmen gegriffen wurde, machte die öffentliche Fürsorge im Nationalsozialismus davon reichlich Gebrauch, bot $\mathbb{2 0} \mathrm{RFV}$ doch eine willkommene Handhabe, um die „Volksgemeinschaft vor schädlichen und sie belastenden Elementen zu schützen“299; seit 1937 übernahmen schließlich Polizei und SS die systematische Bekämpfung der „Arbeitsscheuen“ mit oft tödlichen Folgen für die Betroffenen.

Nichtsdestoweniger wurden die Arbeitshäuser nach 1945 zunächst unter der Obhut ihrer bisherigen Träger weiter betrieben, bis die amerikanische Militärregierung für die US-Zone neben den einschlägigen Bestimmungen des Strafgesetzbuches auch $\int 20$ RFV mit Wirkung vom 1. April 1949 aufhob. ${ }^{300}$ Seit Inkrafttreten des Grundgesetzes galt dieser Paragraph auch in den Ländern der anderen Westzonen als nicht mehr anwendbar. ${ }^{301}$ Die Mehrzahl der Fürsorgeexperten jedoch hielt trotz der Erfahrungen während des Nationalsozialismus den anstaltsmäßigen Arbeitszwang weiterhin für unverzichtbar bei der Bekämpfung von Prostitution, Alkoholismus, der Übertragung von ansteckenden Krankheiten, Nichtseßhaftigkeit oder eben „Arbeitsscheu“302; schließlich, so der NDV im März 1949 in bezeichnend unskrupulöser Diktion, seien diese Bestimmungen ohnehin nur „als letzte Möglichkeit“ tatsächlich angewendet worden, sie „erfüllten ihren Zweck schon dadurch, daß sie als Drohung über den Häuptern dieser sozial labilen Menschen schwebten und sie davon abschreckten, auf dem bisher beschrittenen Weg parasitärer Daseinsgestaltung weiterzugehen ". 303 So standen die fürsorgepolitischen Bemühungen um eine Reaktivierung des $\$ 20 \mathrm{RFV}$ in engem Zusammenhang mit den erneuten Bestrebungen für ein „Bewahrungsgesetz“, die 1961 schließlich in den einschlägigen Bestimmungen der „Hilfe für Gefährdete“ mündeten.

Mit dem „Gesetz über das gerichtliche Verfahren bei Freiheitsentziehung“ vom 29. Juni 1956 wurde zur Befriedigung vieler Fürsorgevertreter u.a. auch die Anwendung des $\ 20$ RFV auf der neuen Grundlage einer richterlichen Einweisung und genauer Verfahrensbestimmungen wieder allgemein ermöglicht und damit die

Ausschreitung mit ihren Folgen“ und zielte damit auf die klassische Klientel der Gefährdetenfürsorge. Vor allem die Einfügung des Schuldbegriffs begründete in seiner $\mathrm{Pa}$ rallele zum Strafrecht den Strafcharakter dieser fürsorgerechtlichen Bestimmung; vgl. Rudolph, Kooperation, S. 74ff.

299 So 1936 die auch nach 1945 einflußreiche Fürsorgepolitikerin und damalige Abteilungsleiterin in der Hamburger Sozialbehörde Käthe Petersen, zitiert nach Rothmaler, Sozialpolitikerin, S. 82.

300 Gesetz Nr.14 der Militärregierung, abgedruckt im Bayerischen GVBl. 1949, S. 78. Danach sollen in der US-Zone mehr als 2000 Personen freigekommen sein; vgl. Ayaß, Arbeitshaus, S. 341, sowie für das Beispiel des hessischen Arbeitshauses Breitenau, ebenda, S. $328 \mathrm{ff}$.

301 Neben Art. 12 Abs. 2 und 3 GG (Zulässigkeit von Zwangsarbeit nur im Rahmen einer allgemeinen Dienstleistungspflicht bzw. bei einer gerichtlichen Anordnung) stand vor allem Art. 104 Abs. 1 GG einer weiteren Anwendung entgegen, wonach die Freiheit der Person nur auf Grund eines förmlichen Gesetzes beschränkt werden darf; die RFV war jedoch kein förmliches Gesetz; vgl. NDV 30 (1950), S. $253 \mathrm{ff}$.

302 Vgl. Kitz, Aufgaben, S. 244ff.

303 NDV 29 (1949), S.37; vgl. auch NDV 30 (1950), S.254; Rudolf Prestel in: NDV 34 (1954), S. 292f. Zu den prononcierten Forderungen nach Wiederbelebung des $\ 20 \mathrm{RFV}$ in Bayern vgl. Rudloff, Schatten, S.361ff. 
Frage seines Einbaus in ein künftiges Fürsorgegesetz akut. ${ }^{304}$ Die Frage, welche Sanktionen im künftigen Fürsorgerecht im Falle beharrlicher Arbeitsverweigerung vorzusehen waren, war natürlich heikel: Einerseits sollte einem partnerschaftlichen, in weite Bevölkerungsgruppen ausgreifenden Hilfeansatz zum Durchbruch verholfen werden, andererseits glaubte man, auch aus Gründen der Gerechtigkeit gegenüber der Allgemeinheit nicht auf Disziplinierungsmaßnahmen verzichten zu können.

Die Fürsorgereformer im Innenministerium suchten den Ausweg von Anfang an in einer weitgehenden Beschränkung dieser Sanktionen auf die klassische Hilfe zum Lebensunterhalt. ${ }^{305}$ Was die Maßregeln selbst anlangte, hielten sich Gottschick und seine Vorgesetzten weitgehend an das bisherige Recht, wobei sie bestehende Unklarheiten zu beseitigen und durch den Einbau sozialpädagogischer Anweisungen den Strafcharakter der Vorschriften teilweise zu mildern suchten. „Unwirtschaftliches Verhalten“ wurde im ersten Referentenentwurf - wohl auch infolge der Ergebnisse der Arbeit am Warenkorb - weniger streng als bisher geahndet. ${ }^{306}$ Wer hingegen zumutbare Arbeit verweigerte, hatte keinen Anspruch auf Hilfe; in dieser eindeutigen Schärfe ging die Sozialabteilung über das bisherige Recht hinaus, denn bislang war es zumindest strittig gewesen, ob die Unterstützung für einen Arbeitsunwilligen ganz versagt werden konnte. ${ }^{307}$ Ausdrücklich untersagt wurde die Einstellung allerdings für den Fall der Schädigung von Unterhaltsberechtigten und Haushaltsmitgliedern - und damit die praktische Anwendbarkeit drastisch eingeschränkt. Mit diesen Vorschlägen bewegte sich die Sozialabteilung auf der Linie, die sich 1957/58 auch im DV und im Beirats-Fürsorgeausschuß herauskristallisiert hatte. ${ }^{308}$

304 BGBl. S. 599. Das Gesetz, das am 1.7.1956 in Kraft trat, setzte in der ehemaligen USZone $\mathbb{} \$ 20 \mathrm{RFV}$ wieder in Kraft und anerkannte diesen vorerst als förmliches Gesetz im Sinne des Art.104 Abs.1 GG. Die Freiheitsentziehung (Arbeitshauseinweisung) war jetzt nur noch möglich durch richterliche Anordnung auf Antrag der zuständigen Verwaltungsbehörde (hier: des Fürsorgeverbands), nicht aber durch diese selbst. Neben Anhörungspflicht und Zulässigkeit der Beschwerde des Betroffenen befristete das Gesetz jetzt einheitlich die Unterbringungsdauer auf höchstens ein Jahr. Das Gesetz galt auch für Freiheitsentziehungen auf der Grundlage anderer Gesetze, war also fürsorgerechtlich relevant bei der Tbc- und der Geschlechtskranken- sowie insbesondere der sog. Gefährdetenfürsorge. Positive Stimmen dazu etwa in: ZfF 8 (1956), S.358f., Petersen, Bedeutung, S. 167f.

305 Vgl. Anlage zur Besprechung mit Abteilungsleiter am 7.2. 1956, BAK, B 106/9688.

306 Laut $\ 30$ des BSHG-Entwurfs 7/1958 sollten „unwirtschaftliche“ Hilfeempfänger jetzt erst einmal „zu einer wirtschaftlichen Lebensführung angehalten werden“; bei Erfolglosigkeit konnte (bisher: mußte) die Unterstützung „auf das zum Lebensunterhalt Unerläßliche" eingeschränkt werden, BAK, B 106/20643.

307 Vgl. $\ 31$ des Entwurfs 7/1958, ebenda. Ebenso wie einer der führenden Kommentatoren, vgl. Jehle, Fürsorgerecht, S. 44, bejahte auch der im DV wiederholt zur Arbeitsfürsorge referierende Offenbacher Jurist Kurth in Anlehnung an einschlägige Gerichtsurteile diese Möglichkeit, vgl. Kurth, Recht, S.203. Die gegenteilige Ansicht bei Zabel, Unterstützungspflicht. Bei Verlust des Rechtsanspruchs war die Hilfe allerdings nicht automatisch einzustellen; vielmehr würde der Träger nach seinem Ermessen über Kürzung, Befristung oder eben Einstellung der Hilfe entscheiden.

308 Vgl. Neuordnung des Fürsorgerechts, S. 216; die Niederschriften über die Sitzungen des Arbeitsausschusses für Fragen der Fürsorge am 4.12.1957, Anlage 1, und am 30./31.1. 
Umstrittener war hingegen, daß der Entwurf auch die Möglichkeit des anstaltsmäßigen Arbeitszwangs für mindestens 18 Jahre alte Arbeitsverweigerer auf der Grundlage des Freiheitsentziehungsgesetzes wieder vorsah. Allerdings entfiel jetzt die explizite Voraussetzung des „sittlichen Verschuldens“, so daß zumindest formal der Strafcharakter der als therapeutisch deklarierten Maßnahme zurücktrat. Nichtsdestoweniger hielten etwa die AWO oder der DV-Fachausschuß für Gefährdeten- und Nichtseßhaftenhilfe wie auch einzelne Ländervertreter die Regelung in einem modernen Fürsorgegesetz für deplaciert, zumal die zwangsweise Anstaltsunterbringung von „Gefährdeten“ künftig im Rahmen der Hilfe in besonderen Lebenslagen gewährleistet schien; eine Regelung zur Abschreckung „Arbeitsscheuer“ hätte allenfalls ihren Platz im Strafrecht ${ }^{309}$ was von führenden Strafrechtlern zum Teil genau umgekehrt gesehen wurde. ${ }^{310}$ Doch trotz der offensichtlich nach wie vor geringen unmittelbaren zahlenmäßigen Bedeutung des $\ 20$ RFV und seiner zahlreichen Anwendungsschwierigkeiten wollten die Fürsorgeverbände wie auch die Sozialabteilung nicht auf die „generalpräventive“ Funktion des fürsorgerechtlichen Arbeitszwangs verzichten. ${ }^{311}$

In der Umarbeitungsphase 1958/59 wurden auf Betreiben der Länderfürsorgereferenten die Schonvorschriften für betroffene Angehörige deutlich zurückgenommen und damit das Drohpotential der Sanktionen wieder erheblich erhöht. ${ }^{312}$ Während dazu von seiten der anderen Bundesressorts keine Einwände erhoben wurden, stieß die Möglichkeit der Freiheitsentziehung hier wie auch bei der Hilfe für Gefährdete auf verfassungsrechtliche Bedenken des Bundesjustizministeriums; es ließ sich aber schließlich durch die Argumentation der Experten für Gefährdetenfürsorge von deren Notwendigkeit auch in einem Fürsorgegesetz überzeugen. ${ }^{313}$ Vor allem die Möglichkeit der Arbeitshausunterbringung nach $\ 26$ verwies damit auf das disziplinierende Element, welches das BSHG von 1961 bei

1958, Anlage 7, ADW, HGSt 6769, sowie des Ständigen Ausschusses für gemeinsame Fragen der Fürsorge und der Arbeitsverwaltung am 14.1.1958, Anlage, ADW, HGSt, SP-S XXIIIc I/O.

309 Vgl. die Äußerungen aus den DV-Fachgremien [5.11.1958], zu \32; Stellungnahme der AWO vom 14.10.1958, BAK, B 106/9686; Niederschrift über die Besprechung mit Vertretern der obersten Ländersozialbehörden am 21./22.10.1958, LAS Abt. 761 Nr. 8874.

310 So der im DV wiederholt als Fachgutachter fungierende Hamburger Strafrechtler Sieverts an Muthesius am 7.5.1958, Abschrift, BAK, B 106/9786/1; tatsächlich sah dann jedoch das reformierte Strafrecht weiterhin eine Arbeitshausunterbringung vor; vgl. Rudolph, Kooperation, S. 84.

311 Vgl. Rudolph, Kooperation, S. 80f.; für die Jahre 1960, 1961 und 1962 (bis 31.5., also kurz vor Inkrafttreten des BSHG) wurden im Bundesgebiet (ohne Saarland und Hamburg) 80 Verfahren nach $\$ 20$ RFV angeordnet. Zu den beschränkten Anwendungsmöglichkeiten (notwendige rechtliche Voraussetzungen, Fehlen entsprechender Anstalten) vgl. Petersen, Bedeutung.

312 Vgl. Niederschrift über die Besprechung am 21./22.10.1958, LAS Abt. 761 Nr.8874; \$S 22f. des BSHG-Entwurfs 3/1959, BAK, B 106/20646; \$S 23f. des Regierungsentwurfs vom Februar 1960, BT, 3. Wp. 1957, Anlagen, Bd.67, Drs. 1799.

313 Vgl. Änderungsvorschläge der Bundesressorts vom 6./8.5.1959, zu $\mathbb{S} \int 22 \mathrm{f}$., BAK, B 106/20647; Vermerk des Referats V 4 zur Rechtsförmlichkeitsprüfung vom 3.10.1959, BAK, B 106/20653. 
aller Modernität auch weiterhin enthielt. ${ }^{314}$ Die nach wie vor virulente Frage ihrer Verfassungsmäßigkeit wurde in der überwiegenden zeitgenössischen Rechtsmeinung wie auch vom Bundesverfassungsgericht noch 1970 ausdrücklich bejaht, obwohl zu diesem Zeitpunkt bereits die entsprechenden Bestimmungen der Gefährdetenhilfe für verfassungswidrig erklärt worden waren. Wenn $\mathbb{2} 26$ schließlich im Rahmen der BSHG-Novellierung 1974 doch aufgehoben wurde, dann lag es primär daran, daß mangelnde Möglichkeiten und hohe Kosten der Unterbringung deren Abschreckungsfunktion obsolet gemacht hatten. ${ }^{315}$ Nach Aufhebung der strafrechtlichen Arbeitshausmaßregel 1969 war nun auch die Arbeitshaustradition im bundesdeutschen Fürsorgerecht endgültig beendet.

314 Vgl. auch Barabas/Sachße, Bundessozialhilfegesetz, S. 372f.

315 Vgl. Rudolph, Kooperation, S. 84ff.; Ayaß, Arbeitshaus, S. $342 \mathrm{ff}$. 